\title{
PERSISTENCE OF EMBRYONIC FIBROVASCULAR SHEATH OF THE CRYSTALLINE LENS.
}

\author{
Edwin J. Lent, M.D., and Martha Brewer Lyon, M.D. \\ SOUTH BEND, INDIANA.
}

The published cases of this condition are here abstracted and reviewed, and two new cases are reported. Its differential diagnosis is discussed. This is important because eyes presenting this anomaly have been enucleated on the supposition they were affected with glioma of the retina. A bibljography is appended. Rcad before the Indiana Academy of Ophthalmology and Oto-Laryngology at Indianapolis, January 19, 1922.

This paper was suggested by an unusual condition found in a patient who came for relief from poor vision, by the small number of similar cases reported in the litcrature, and by the possibility of such cases being mistaken for other conditions, especially glioma, unless a careful diagnosis is made.

The embryonic blood supply of the capsule of the lens, according to accounts of Gradle and of Fuchs, shows the following groups of blood vessels, (1) Those given off from the central artery of the optic nerve, which continues as the hyaloid artery toward the posterior pole of the lens, in Cloquet's canal, halfway thru the vitreous chamber, where it separates into 8 or 10 branches of equal size, which spread peripherally in a meshed formation anastomosing frecly with one another, and enter the lenticular sheath midway between the equator and the posterior pole of the lens. Each of these branches subdivides rapidly, making of the lenticular sheath a meshwork of fine arteries, which are interspersed by many capillaries radiating unequally in all directions. (2) The central artery of the optic nerve as it enters the eye gives off lateral branches which form an arterial network in the peripheral portions of the vitreous, and extend forward to the margin of the lens, uniting and anastomosing with branches from the hyaloid artery, forming a specially dense network of vessels surrounding the border of the lens.

The anterior branches of the long ciliary arteries, which course thru the mesoderm that covers the surface of the iris, bend over the pupillary edge of the iris and assist in covering the anterior capsule of the lens with a vascular network. The course of these vessels as they pass thru the vascular sheath, over the anterior surface of the lens, is not radial, but assumes a finely woven meshwork anastomosing freely with one another. (4) The veins are derived from the circulation of the iris, and are found slightly posterior to, and directed toward the equator of the lens; and provide for the escape of all the blood into the ciliary region and ultimately into the venae vorticosae.

Two areas of the entire lens surface, the anterior and posterior poles, remain comparatively free from vascularization.

The hyaloid system and the tunica lentis vasculosa reach the height of their development during the sixth month of intrauterine life, while in the sixth and first part of the seventh months, the disappearance of the entire hyaloid system occurs by rapid absorption of the vessels accompanied by an equally rapid disappearance of the tunica lentis vasculosa. Failure of the arteria hyaloidea to be absorbed gives the anomaly known as the persistent stump of the hyaloid artery, while permanent remnants of the tunica lentis vasculosa are known as pupillary membrane.

An endeavor has been made to examine the literature pertaining to all the anomalies found in connection with the embryonic blood supply of the fibrovascular sheath of the lens.

The earliest mention of the vascularity of the lens and its capsule is said to have been published by Howard in 1851. DeWecker observed, in an eye with upward dislocation of the lens, an atrophied artery which extended from the papilla forward and upward, and which terminated in a white substance surrounding the lens. He also described an interesting case of persistent canal of Cloquet, terminating in the shape of an $S$ at the posterior capsule 
of the lens; but he did not mention a ramification of blood vessels over the lens.

Fournet in 1879 reported a case of persistent hyaloid artery in a soldier 22 years old with external strabismus, who came into the hospital suffering with pneumonia.

Gardiner in 1880 reported a case of persistent hyaloid canal and artery in the right eye of an American, 22 years old, who consulted him for dimness of vision. Extending from the papilla forward to the posterior capsule of the lens, was a white shining cord of about the caliber of one of the principal branches of the retinal artery. At the insertion into the optic nerve, it formed a funnel shaped expansion entirely covering the papilla and its vessels, and extending beyond at the nasal side. The anterior portion formed a similar smaller funnel, attached to the posterior capsule of the lens;--the cord exhibited rhythmic movements and was found to consist of a bright red blood vessel enveloped in a thin translucent sheath, Cloquet's canal. The expansion at the posterior capsule consisted of numerous little blood vessels spreading and ramifying over the posterior polar region of the capsule. Details of fundus could not be made out very well, and the vitreous was somewhat hazy. There were no traces of similar condition in the left eye. Under atropin, vision right eye $20 / 200$, unimproved by lenses; vision left eye $15 / 200$ improved to $20 / 40$, by plus 12.00 sphere.

Holmes in 1881 reported a case of persistent hyaloid canal in an amblyopic right eye, with strabismus, in a child of 17. Vision 5/100. Under atropin the pupil, which was sluggish, dilated only moderately. A central defect in the field of vision was caused by a white object, shaped like a wine bottle with base on the optic disc, with body, neck and head projecting forward almost to the center of the posterior capsule of the lens. At first it was taken for a cysticercus. No drawings published.

Snell in 1883 reported a case of posterior polar opacity of the lens and persistent hyaloid artery in the left eye, in a child of 11 years, with vision of fingers at 1 foot, and a myopia of $8 \mathrm{D}$. The opacity in the posterior pole of the lens was made up of fine striae in the posterior capsule. Running backwards from the opacity was a light grey translucent hollow cord. attached at the optic disc to a vessel in the center of the papilla, widening out like a funnel, wavy between attachments. No illustration given.

Collins in 1892 gave a detailed anatomic description of the persistent posterior fibrovascular sheath of the lens in three cases, and subsequently has recognized a like number of cases clinically. Collins is quoted by de Schweinitz in 1899 as saying "persistence of the posterior part of the fetal fibrovascular sheath of the lens may be mistaken for glioma."

Brailey and Ormond in 1899-1900 reported a case of probable congenital vascularity of lens capsule, in the right cye of a child $21 / 2$ years old. This abnormality was commented upon at its birth by the attending doctor. The mother noticed it "blood shot." At 1 year the child developed an occasional squint. An opacity was found occupying the inner $2 / 3$ of the anterior part of the lens, while the outer $1 / 3$ was almost clear, with some small scattered opaque spots. On the innermost part of the opaque area were several small vessels with minute branches. The opacity was chalky white and had an uneven surface, the pupil eccentric, the iris, broader at outer than at inner side, dilated only moderately under atropin. No illustration given.

Silcock in 1900 reported a vestigial hyaloid artery starting from a macular coloboma in a child of 16 years who complained of short sight in left eye. From the center of a circular macular choroidal coloboma, a connective tissue thread like branch extended forward thru the vitreous, terminating in a fimbriated extremity, which was attached by a thin strand of connective tissue to the posterior surface of the lens capsule, at the lower and inner part.

Middleton in 1919, out of 28 cases of congenital condition of the fundus or 
bulb causing a reduction of vision, observed in the examination of 200,000 soldiers, reported one case of complete pupillary membrane, with slight dimness of vision in the right eye, altho vision was stated to be $20 / 20$. The iris was free from adhesions, but there was a thin, congenital, film like membrane nearly transparent, covering the entire right pupil and attached to the iris equally around the whole of the pupil. Examination with a loupe showed a vertical slit $2 \mathrm{~mm}$. wide at center, extending across the pupil, resembling the slit like pupil of a cat's eye, and gradually coming together at each end near the margin of the pupil. Atropin dilated the pupil slightly horizontally but not vertically, while the opening in the pupillary membrane was widened to twice its original width. The distant vision was reduced under atropin to $20 / 200$. The left pupil was free from membrane or pupillary bands. Vision in left eye $20 / 30$.

Lane in 1919 reported two clinical cases, and three eyeballs examined microscopically, with persistent posterior fibrovascular sheath of the lens.

Of the clinical cases, one a child of 14 years, had convergent upward squint since one year of age, with vision in right eye $3 / 200$. Right lens in anterior nasal third of cortex was irregularly opaque at different levels. Opacity continued to the mesial side and involved larger area of posterior cortex. A chalk like opacity was nasally displaced on the vitreous surface of posterior capsule. A long narrow vertical slit thru this opacity showed a red reflex, and from the opacity 5 or 6 narrow, spindle, grayish white striae ran forward and obliquely downward in the nasal direction. $A$ reddish hyaloid artery extended from disc to lens.

The second child, 6 years old, had poor vision and white pupil in the right eyc. Vision was limited to shadows, no red reflex. The pupillary and ciliary zones of iris were not definitely demarcated, and a network of delicate pupillary membrane threads stretched across the lower third of right pupil. Under atropin pupil dilated irregularly below, where three short narrow tri- angular shaped tags of iris appeared to be pulled toward the center by pupillary threads. Completely surrounding the posterior surface of the lens was a glistening uniformly white opacity, on the anterior surface of which was a single tortuous vessel running across the horizontal meridian. Four or five finer vessels taking a horizontal course were below, toward the temporal side. Left eye, vision limited to fingers at 18 inches. Left pupil dilates regularly. An opacity lay back of the upper nasal quadrant and spread out behind the upper one-fourth posterior surface, as a fixed cobweb like network. No fundus details were made out. Refractive error was about plus 6 .

The three eyes examined microscopically by Lane were enucleated for suspected glioma. One, a baby of $1 \cdot 1 / 2$ years with a gray spot behind the left pupil seen by his parents, showed a vascularized white tissue behind the lens, which proved, after the eye was removed, to be a persistent posterior fibrovascular sheath of the lens with patent hyaloid artery attached. In the second baby, when 2 months old, was noticed a discolored pupil in the right eye. At 3 months there was a shallow anterior chamber and a reddish gray reflex, behind the lens. Microscopic examination showed a persistence of pupillary membrane, a persistent fibrovascular sheath of the lens, with a gap in the posterior capsule, hemorrhage in lens and a hyaloid artery. In the third baby, 3 months old, the left lens was seen to be pushed forward on the nasal side, and a gray vascular tissue was situated in the most anterior region of the vitreous. In the removed eye was found a persistent posterior fibrovascular sheath of the lens, with hyaloid artery and a partial irregular development of lens.

More recently several cases were presented before different socicties, but illustrations were not published and letters from most of the authors showed none had been made. Schwenk's case was that of a Russian girl, 15 years old, who had poor vision, pain in both eyes after reading and excessive lacrimation. Under a mydri- 
atic pupils were irregular. Right eye: Three distinct brown bands were attached to the smaller circle of iris and, crossing the pupil to the opposite side, were adherent to the lens capsule in the pupillary area, appearing like a brown membrane on the lens, almost covering the pupillary area. Vision 20/200, improved to $20 / 70$ by minus 2.50 sphere. Left eye: Four bands crossing from side to side nearly at right angles gave a brownish membrane appearance on lens capsule. Vision 20/70, improved to $20 / 50$ by minus 3.00 sphere.

Shields' patient was a man, 28 years old, born in Russia. Two weeks previous to consultation, he discovered that vision in left eye was practically gone. On the anterior capsule of both lenses was a deposit of minute pigmentary areas, more dense in left than in right eye. In left cye was a fragment of brownish tissue at the margin, which appeared to be a tag of pupillary membrane. Vision left eye, fingers at two feet.

Crisp reported a case of persistent remains of the embryonic system of blood vesscls, between the optic disc and the lens in the right eye of a woman of 29 years. Vision, right eye with correction of plus $3.00 \mathrm{~S}$. is $5 / 10$ plus; left cye with plus $1.25 \mathrm{~S}$. is $5 / 3$. The remains appeared as a black strand, wavy and fairly mobile, extending completely from disc to posterior pole of lens.

Suker presented a young man with a persistent hyaloid artery and a fusiform aneurysm of the superior temporal retinal artery in one eye. There were three distinct motile branches, 3-5 $\mathrm{mm}$. in length, on the temporal side of the disc, attached to the main trunk of the remains of the hyaloid artery, which was fixed and $5 \mathrm{~mm}$. long. On the superior temporal retinal artery were seen several fusiform dilatations about $5 \mathrm{~mm}$. long. Vision, normal.

In recently abstracted literature, three additional cases are cited. 'Takao saw a patient with pupillary membranes in both eyes. The membrane was on a plane with the anterior surface of the iris, with an opening in the middle in front of the true pupil.
Axenfeld reported a case of movable pupillary membrane in a young man having diminished vision in a bright light. Pupils were slightly irregular, with a greyish color in the pupillary areas, which disappeared on looking up. Lateral illumination showed a pupillary membrane thrown into folds when contracted, which became smooth and transparent on dilatation of pupil. The membrane was attached to the posterior surface of iris.

Perry had a case with normal vision, in which a remnant of the hyaloid artery was attached to posterior capsule of the lens and ended in the vitreous.

Rumbaur in 1921 described star shaped remnants of the pupillary membrane in the anterior lens capsule. These could be seen under magnification of 60 or 70 with strong illumination. According to Rumbaur, the star shaped formations occur in $35 \%$ to $40 \%$ of all individuals, of which $2 / 3$ show the anomaly in both eyes, and in persons of all ages, 5 years to 72 years, decreasing in frequency with increasing age. They occur with other anomalies found at birth and often with other remnants of pupillary membrane, with which they seldom stand in direct relation. Defective vision on account of them seldom occurs, because of their peripheral location. He also gives references to several articles in German literature dealing with pupillary membrane. These have not been seen.

\section{REPORT OF CASES.}

CASE 1. Mary H., aged 7 , referred May 5, 1921, by school nurse, because of inability to see blackboard.

Vision in each eye $20 / 100$, not improved by lenses. Retinoscopic examination under homatropin cycloplegia, alone or with minus or plus lenses, failed to show movement of shadow across the pupil, altho the red reflex was quite distinct. The trial case test gave no improvement in vision. The ophthalmoscope revealed the following picture. Fundus in either eye seen with difficulty, but apparently normal.

On examination of the right eye, with plus 20 behind the aperture, a 
fine meshwork of thread like lines appeared to cover the central part of the lens, with an occasional small nest like terminal dot surrounded by meshwork. In the left eye, with a plus 20 behind the aperture, a similar meshwork was seen, but with larger and more prominent, dark, nest like spots and larger portions of the lens clear. No remnants of hyaloid artery could be seen in either eye. A $1 / 2 \%$ atropin

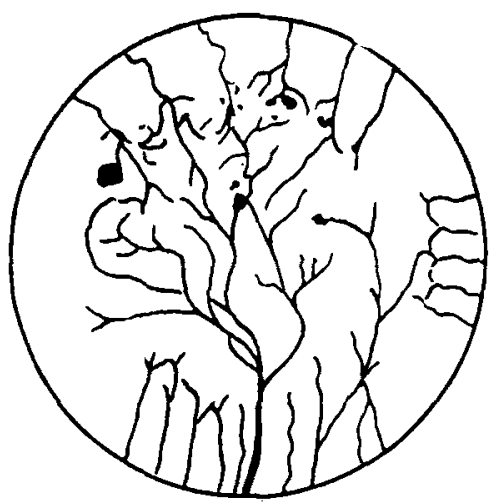

Fig. 1. Appearance of blood vessels in fibrovascular sheath of lens of right eye of case 1 described in text.

solution was instilled three times a day for four successive days, at home, and again an effort was made to improve vision from the trial case, but without success.

The complete dilatation of the pupils showed additional thread like meshwork around the periphery of the lens in either eye, which had not been scen before. In either eye the central meshwork seemed to branch from a main stem, which was thickest at the lower edge of the lens. In and surrounded by the meshwork, were the small round nest like spots, more prominent in the left eye. The fine meshwork around the periphery of the lenses seemed to have no connection with the larger central masses, and did not reach more than one-fourth distance across the lens. Drawings were made with the child in the recumbent position, looking up at the ceiling or fixing a finger held two feet directly above one eye. Nothing of importance in the family history could be obtained. The patient is the oldest of three children and the only one unable to see well. The mother and one younger child were examined and had apparently normal eyes. The father and other child could not be obtained for examination.

Case 2. Mírs. C. brought her small daughter because of -impaired vision. History: The mother, had an undeveloped right eye, a defect which she feared might have been transmitted to her child. Examination of this eye

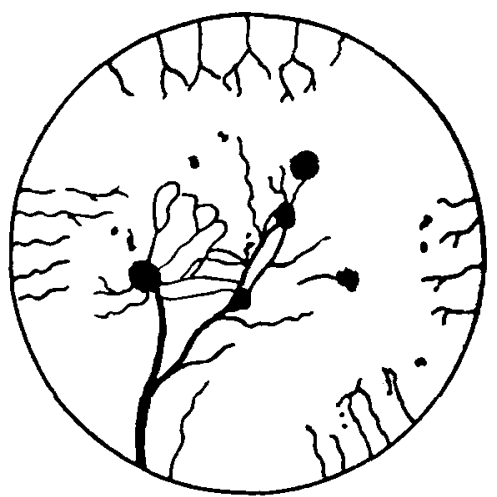

Fig. 2. Appearance of blood vessels in fibrovascular sheath of lens of left eye of case 1 described in text.

under $4 \%$ cocain mydriasis showed a marked opaque fibrovascular sheath, with minute blood vessels radiating from it. She stated this was noticed at birth. During infancy and childhood, the eye was carefully watched for inflammatory symptoms and blindness. She has peripheral vision of large objects. She could not be persuaded to sit for a drawing of the anomaly. DIFFERENTIAL DIAGNOSIS.

Brief differential diagnosis between glioma of the retina and persistent fibrovascular sheath, and between cysticercus of the vitreous and either persistent hyaloid artery or persistent fibrovascular sheath, may not be out of place.

Glioma of the retina occurs most frequently in children, usually under five years, most often involving one eye, altho bilateral cases have been reported. There is noticed first, a bright, whitish or golden yellow reflex from the pupil and a lack of vision. Under focal illumination a light colored nodular mass behind the lens covered with 
minute vessels is seen. At first there are no inflammatory symptoms, only the light colored reflex and the blinding of the eye. It is at this stage that a persistent fibrovascular sheath may easily be mistaken for it.

As gliomas grow rapidly and persistent fibrovascular sheaths remain stationary, careful measurements will determine the diagnosis. Glioma, if seen early, when it is small, is visible thru the naked aperture of the ophthalmoscope, while a fibrovascular sheath requires a plus 16 to plus 20 lens to bring out the remnants of the minute blood vessels connected with it. These two points alone would serve to differentiate between glioma and persistent fibrovascular sheath.

Hyaloid artery with a funnel shaped extremity with its rythmic movements may be mistaken for cysticercus in the vitreous, as in Holmes' case. The parasite appears as a bluish white, bottle shaped bladder, if head and neck are extended, or as a brilliant white spot, if head and neck are withdrawn. The head in early cases moves actively. If this is extended, there may be seen one of the four suctorial discs and a crown of from 2 to 20 hooklets. If of long duration, opacities of the vitreous form around it, and it appears as a dense white mass. At this time it may be mistaken for a persistent fibrovascular sheath. In cysticercus infestation vision is finally lost; but in persistent fibrovascular sheath vision, altho reduced, remains unchanged. The. diagnosis of a cysticercus from a persistent hyaloid artery can be made by the hooklets on a moving head; while the persistent fibrovascular sheath may be diagnosed by the strength of the lens necessary to see the latter, the amount of vision remaining and $a b$ sence of inflammatory symptoms.

In closing we would suggest a systematic reporting of all similar cases, with drawings if possible, that a knowledge of their prevalence may be obtained; that a more careful differential diagnosis be made to eliminate the tragedy of enucleation in questionable glioma and cysticercus, and a more detailed study of lens capsule in both diseased and normal conditions along lines suggested by Rumbaur.

\section{BIBLIOGRAPHY.}

Brailey, W. A., and Ormond, A. W. Vascularity of lens capsule probably congenital. Trans. Ophth. Soc. United Kingdom, 20, 187, 1899-1900.

Crisp, W. H. Remains of hyaloid vessels. A mer. Journ. Ophth., 3, 377, 1920.

Fournet, F. De la persistance du cordon hyaloidien. Rec. d'Opht., ser. 1, 3, 522, 1879.

Fuchs, E. Text-book of Ophthalmology, 11 ed. Trans., A. Duane, 393, 1917.

Gardiner, E. J. Persistent hyaloid canal and artery. Arch. Ophth, 9, 473, 1880.

Gradle, H. S. Development of the human eye. Amer. Enc. Ophth., 5, 3915, 1914.

Holmes, E. L. Case of persistent hyaloid canal. Arch. Ophth., 10, 168, 1881.

Howard, H. Further proof of the vascularity of the lens and its capsule. Brit.-Amer. Journ. Med. and Phys. Sci., 7, 150-153, 1851-2. Cited in Index-Cat. Libr. Surg.-Gen. Off. U. S. Army, 8, 27, 1887.

Lane, Francis. Persistent posterior fibrovascular sheath of the lens. A report of two clinical cases, and three eyeballs examined microscopically. Arch. of Ophth., 48, $572-580,1919$.

Middleton, A. B. Congenital eye conditions affecting vision out of 200,000 troops examined at Camp Travis, Texas. Amer. Journ. Ophth., 2, 377-388, June, 1919.

Perry, T. E. Persistent hyaloid artery. West Virginia Med. Journ., 14, 450, 1920. Cited in Ophth. Lit., 17, 216, June, 1921.

Rumbaur, W. Klin. $M_{b}$ f. Augenh., 66, 737-742, 1921.

Schweinitz, G. E. de. Diseases of the Eye, 3 ed., 465, 1899.

Schwenk, P. N. K Persistent pupillary membrane. Amer. Journ. Ophth., 3, 295, 1920.

Shields, J. M. Dust like persistent pupillary membrane. Amer. Journ. Ophth., 3, 377, 1920.

Silcock, A. A. Vestigial hyaloid artery starting from a macular coloboma. Trans. Ophth. Soc. United Kingdom, 20, 188, 1899-1900.

Snell, S. Posterior polar opacity of lens and persistent hyaloid artery, Trans. Ophth. Soc. United Kingdom, 4, 349, 1883-4.

Suker, G. F. Persistent hyaloid artery and fusiform aneurysm of superior temporal artery in same eye. Amer. Journ. Ophth., 3, 534, 1920.

Takno, J. Pupillary membrane with pseudopupil. Nippon Gank. Zasshi, Oct., 1919. Cited in Ophth. Lit., 17, 134, June, 1921.

Wecker, de. Artere hyaloide persistante avec cataracte luxee, Annales d'Oculistique, 53, 65.

Wecker, de. Traite des maladies des yeux, 48. Cited by F. J. Gardiner, Arch. of Ophth., 9 , 474,1880 . 